\title{
A Portable Elbow Exoskeleton for Three Stages of Rehabilitation
}

\author{
Soumya K Manna, Venketesh N. Dubey \\ Faculty of Science and Technology, Bournemouth University \\ Talbot Campus, Poole, BH12 5BB, United Kingdom
}

\begin{abstract}
Patients suffering from stroke need to undergo a standard and intensive rehabilitation therapy. The rehabilitation training consists of three sequential stages; the first stage is controlled joint movement under external actuator, the second stage deals with supporting the movements by providing assistive force and the last stage provides variety and difficulty to exercises. Most of the exoskeletons developed so far for rehabilitation are restricted to a particular type of activity. Although a few exoskeletons incorporate different modes of rehabilitation, those are software controlled requiring sensory data acquisition and complex control architecture. To bridge this gap, a portable elbow exoskeleton has been developed for delivering three stages of rehabilitation in a single structure without affecting the range of motion and safety features. Use of electric motor and springs have been arranged in the actuation mechanism to minimise the energy consumption. The developed exoskeleton enhances torque to weight ratio compared to existing models and all three modes of rehabilitation have been controlled using a single motor.
\end{abstract}

Keywords: Exoskeleton, Rehabilitation, Portable, Stiffness control, Gravity compensation, Stroke.

\section{Introduction}

Present statistics shows that there are about 33 million stroke survivors worldwide [1]. The annual health and social costs of caring for disabled stroke patients are estimated to be in excess of $£ 5$ billion in the UK alone [2]. To reduce the burden of manual therapy which includes unavailability of sufficient number of caregivers and intensity of exercises, exoskeleton based rehabilitation has become a promising alternative [3]. However, the majority of developed exoskeletons can provide only a specific type of exercise [4]. A few standard rehabilitation processes are followed from acute stage to full recovery stage after stroke [5], [6], [7]. All these stages are used to regain the controlled muscle movement by reducing spasticity and involuntary movement. After analysing the function and treatment procedure of each stage, these can be categorized into three distinct stages as shown in Fig. 1. These are actuator based joint control, supportive force and resistive force.

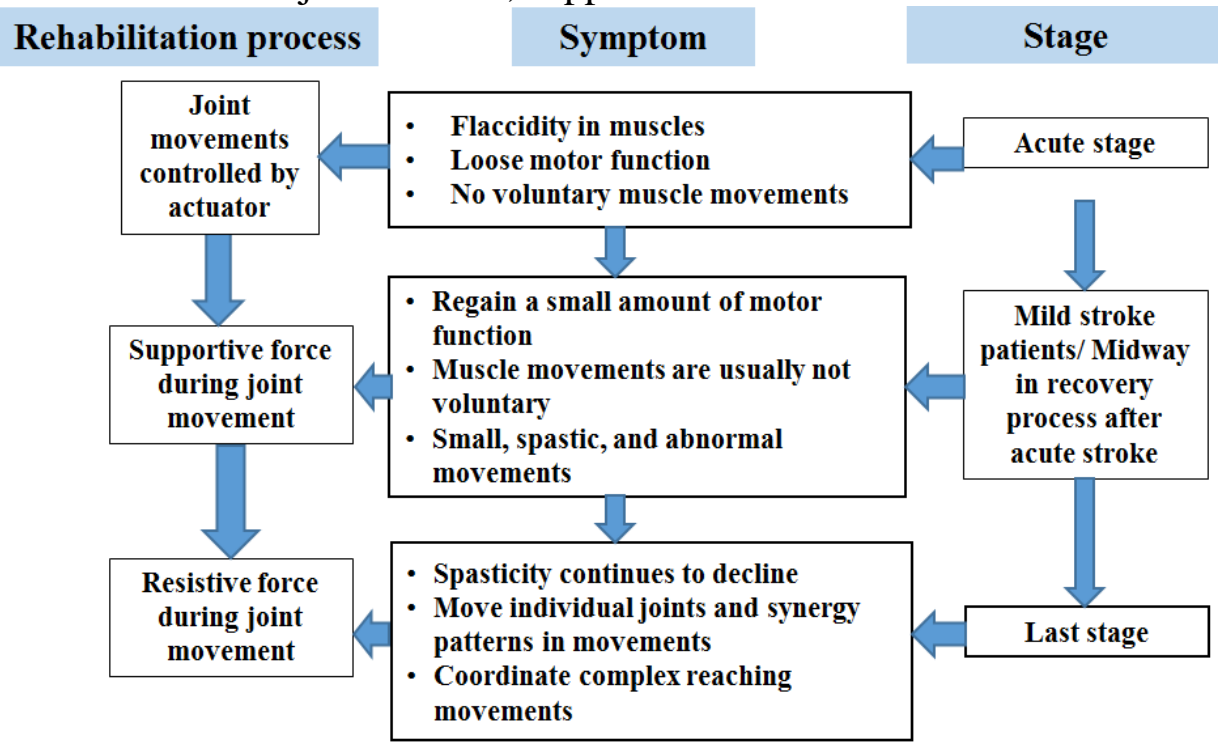

Figure 1. Three phases of rehabilitation for post-stroke patients

If exercise is performed in transverse plane using exoskeleton, patients only need to overcome the frictional force of the exoskeleton structure. If the same exercise is performed in sagittal plane, the 
exoskeleton requires higher joint torque to carry out the load against gravity; size of the actuator as well as the actuation system needs to be effective to obtain the required torque. The range of movement is improved if exercises were performed in gravity compensated training environment [8]. Postural stability is achieved by active holding of the body segment against external force [9].

Even after three decades of research, no standard solution has been presented for the design of exoskeleton to provide the best rehabilitation therapy [10]. Most of the exoskeletons have focused on design aspect which includes portability and user-friendliness but failing on providing the standard rehabilitation training. Two essential factors can be considered for the design of exoskeletons; one is its mechanical design and other is the rehabilitation therapy.

The quality of rehabilitation therapy using exoskeleton can be improved by introducing three stages of rehabilitation in a single structure which can possibly be accomplished in two ways; one is the hardware-based solution and the other is software approach. In software approach [11], actuator used in the exoskeleton can provide three stages of therapy using adaptive control algorithms. In order to execute patient-oriented exercises using external actuators, electric motors are normally placed at the joint of most exoskeletons [12]. Here different bio-sensors attached to user send signals to the control system about the patient's intention and the motors provide assistive or resistive torque to the affected joint as per the signal received from biosensors (EMG, EEG). Because of the reliance on biosignals, those systems are inoperable without sensors. Movement based on EMG data extraction from stroke patients is difficult because of abnormal EMG-torque relationship [13]. The adaptive control algorithm used in exoskeleton results in constant draining of energy for controlling the variable joint torque and active range of motion. Also, the adaptive control system always takes over the control by making patients inactive which indirectly reduces their activities during training [14], therefore diminishes the rehabilitation effectiveness. Joint-based actuation system also requires higher torque compared to the designs where joint is remotely controlled. To carry out the exercises with higher load, size and weight of the motor are also increased [15] and so is the cost. As a result, most of the electric motor controlled exoskeletons are ground-based system [16]. In the former case, human joint is always under motor control which might not be ideal from safety point of view. If the joint moves beyond the anatomical range, it may cause injury. Looking at these limitations, a hardware-based solution may provide viable option for user acceptance.

Integration of multistage rehabilitation can be achieved using active and passive components in the mechanism which can reduce the complexity of the control system. For example, a system can use an electromagnetic clutch/brake for shifting from one rehabilitation mode to another though it will drain energy and create unwanted noise during switching. Passive actuation systems use elastic elements such as spring or rubber band which can provide the required joint torque for reducing the gravity force during elbow movement. Such spring-based exoskeletons [17],[18] do not need any energy source to actuate but these systems can only provide assistive force to users. The backdrivable motor in combination with a series elastic actuator [19] is also able to provide both types of rehabilitation, however if the back-drivability is too low, the gearbox can be damaged due to sudden external force. Compliant mechanism [20] can provide variable stiffness to the joint however, it can only generate resistive force.

Hence it is quite challenging to integrate all types of exercise in a single exoskeleton because exercises involved in the three stages after stroke are totally different in nature. In acute stage, patients require fixed contact to human arm because they have no power left to move their arm but in rest of the recovery stages, exoskeleton needs a compliant contact to allow them to carry out the exercises themselves. The aim of this paper is to create an innovative joint mechanism for the exoskeleton to achieve both these properties without any extra burden to system or risk to the users. The novelty of the developed exoskeleton can be described as:

- The developed exoskeleton delivers all modes of exercises (external force, assistive and resistive) required for three stages of rehabilitation in a single structure.

- The exoskeleton mechanism generates variable assistive as well as resistive force without using any complex control algorithms. 
- Electric motor is used to control the joint whereas in rest of the two modes, joint motions are supported by stiffness of the springs for providing assistive or resistive force.

- Spring stiffness is used for the switching mechanism to shift between rehabilitation modes, therefore, no brakes or clutches are required making it an energy efficient mechanism.

- The switching mechanism supports safety of the users mechanically since joint control is transferred from motor to user when the elbow joint goes beyond the permissible limit.

- To achieve all this a single motor has been used in the whole exoskeleton design.

\section{Design description}

The exoskeleton has been conceptualized as a mechanism where the whole operating region is divided into three sub-regions to provide specific exercises as shown in Fig. 2. All these regions are interconnected and will appear one after another mechanically. The type of exercise generated by exoskeleton is aligned with the post-stroke recovery stages. The exoskeleton utilizes the motor torque in acute phase when users do not have enough strength and provides spring energy during self-movement for generating assistive and resistive force. A couple of springs (compression and torsional) have been used in the exoskeleton for switching between different regions. The schematic diagram of the exoskeleton and its 3D model are shown in Fig. 3 (a and b).

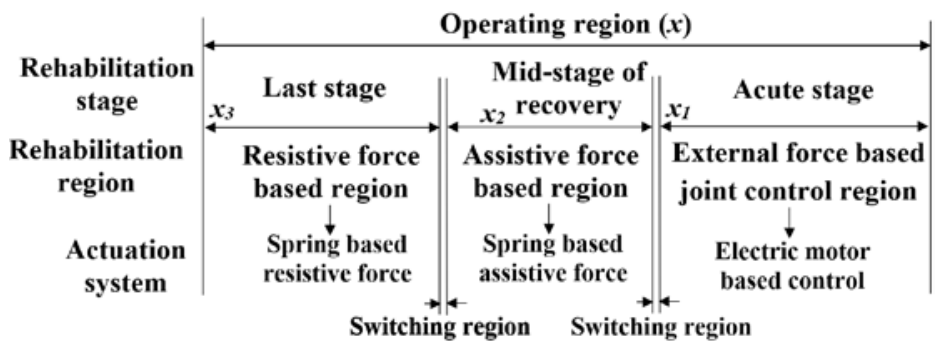

Figure 2. Operating region of the exoskeleton

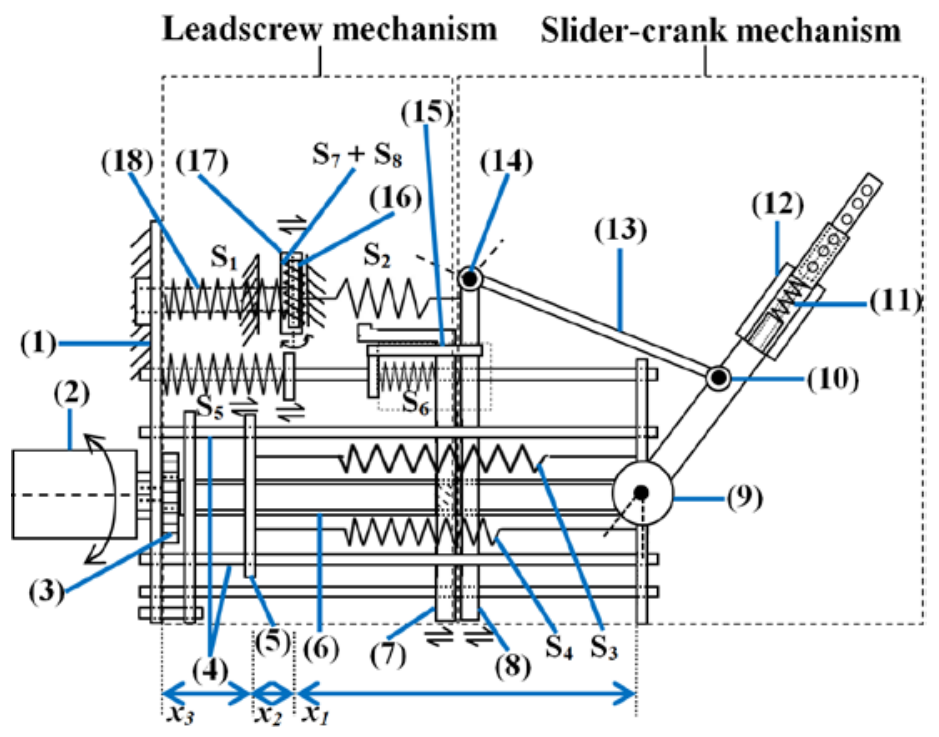

(a) Schematic diagram 


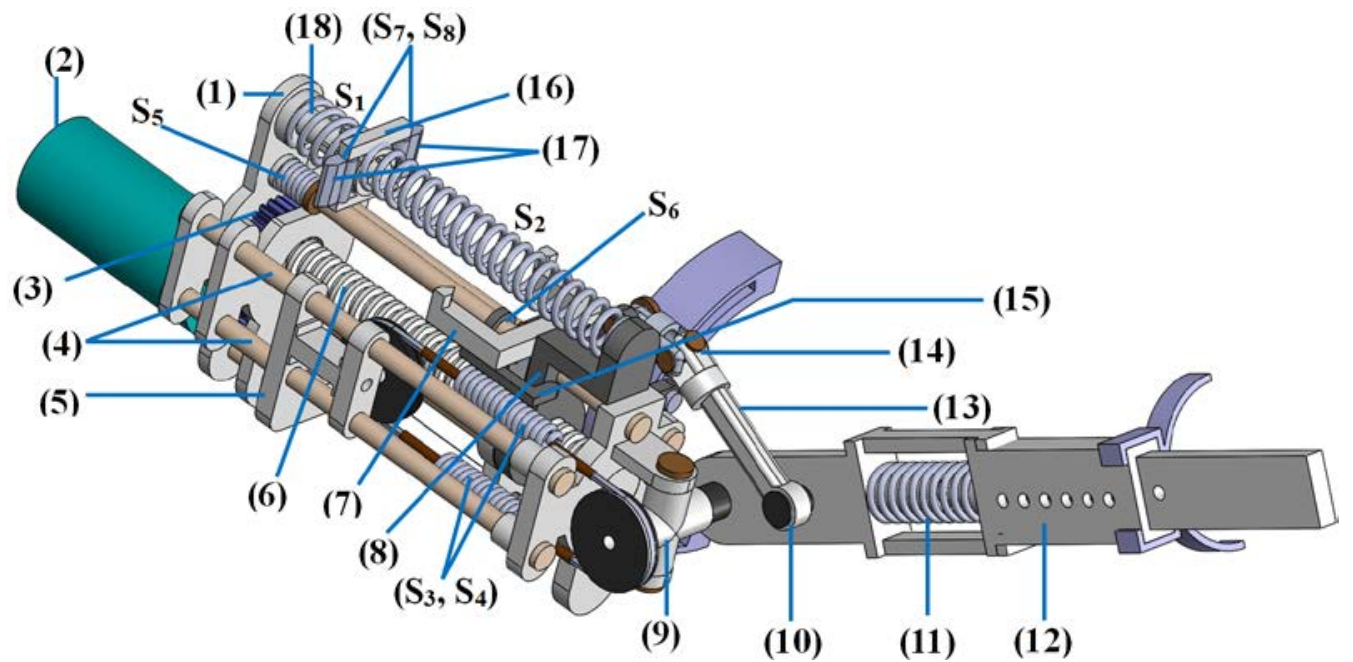

(b) 3D model

Figure 3. Exoskeleton Design
(1) Baseplate
(7) Nut slider
(8) Concentric slider
(13) Connecting link
(2) Motor
(9) Elbow joint
(3) Gear
(4) Solid rods
(10) Revolute joint
(5)Slider for variable stiffness
(11) Compression spring
(12) Forearm supporting link
(14) Universal joint
(15) Claw-type jaws
(16) Rectangular slider
(17) Connected plates
(18) Small cylindrical rod

The relationship between the distance covered by the nut slider $(x)$ and the motor rotations $(n, \theta)$ is given by

$x=\frac{n \theta L}{2 \pi N}$

Where, $n$ - Number of turns of the motor

$\theta$ - Angle made by the motor

$L$ - Lead of the screw

$N$ - Gear ratio for transferring the motion to the leadscrew $(1.5: 1)$

Mode of rehabilitation: $\quad 0 \leq x \leq x_{1} \quad$ = Electric motor based joint control ( 0 to $\left.0.18 \mathrm{~m}\right)$

$x_{1}<x \leq x_{2}=$ Spring based assistive force $(0.181$ to $0.20 \mathrm{~m})$

$x_{2}<x \leq x_{3}=$ Spring based resistive force $(0.201$ to $0.24 \mathrm{~m})$

Where $x_{1}, x_{2}, x_{3}$ are the switching positions.

\subsection{Electric motor based joint control (first region)}

In the first region, the electric motor controls the joint movement without any active participation from the user. The actuation system has been designed based on a leadscrew in combination with a slider-crank mechanism (Fig. 4). Motion from the motor is transferred to the leadscrew through reduction gears. Slider-crank mechanism converts the linear motion of leadscrew into elbow joint rotation. The leadscrew and slider crank mechanism are not directly coupled to each other. There are two loads to overcome; one of which acts as a nut that translates in both directions on the screw and the other slider moves over the leadscrew concentrically without being placed on the leadscrew. In the first region $\left(0<x \leq x_{1}\right)$, a spring $\left(\mathrm{S}_{6}\right)$ actuates the locking mechanism that keeps both sliders in contact as a single unit until the elbow joint rotates to its maximum anatomical limit $\left(0-130^{\circ}\right)$. 

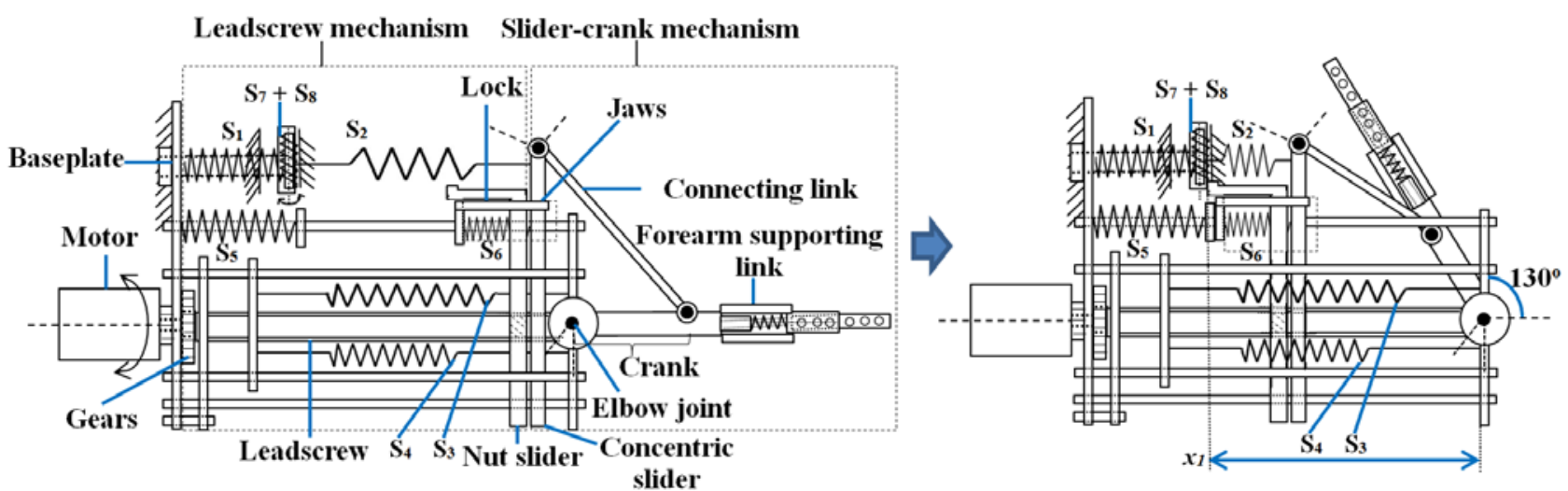

Figure 4. Electric motor based joint control

\subsection{Switching from the electric motor control (first region) to assistive force (Second region)}

In the locking mechanism, two claw-type jaws are connected to the nut slider in the form of a fourbar mechanism (Fig. 5(a), Locked condition). The locking condition remains enforced until the two compression springs $S_{5}$ and $S_{6}$ clash with each other due to the backward movement of the nut slider. As soon as the nut slider crosses the switching position $\left(x>x_{1}\right)$, switching takes place. Because of the higher stiffness, the force exerted by $S_{5}$ is greater than $S_{6}$, thus a small displacement of $S_{5}$ causes a large displacement in $\mathrm{S}_{6}$. As a result, $\mathrm{S}_{6}$ will be compressed by the resultant force and both jaws will rotate about a fixed point to free those sliders (see the unlocked condition, Fig. 5(b)). However, forward movement of the nut slider beyond the switching point will restore the locking mechanism again. The ratio of the stiffness of $S_{5}$ and $S_{6}$ has been determined in a way that the switching region becomes as small as possible.

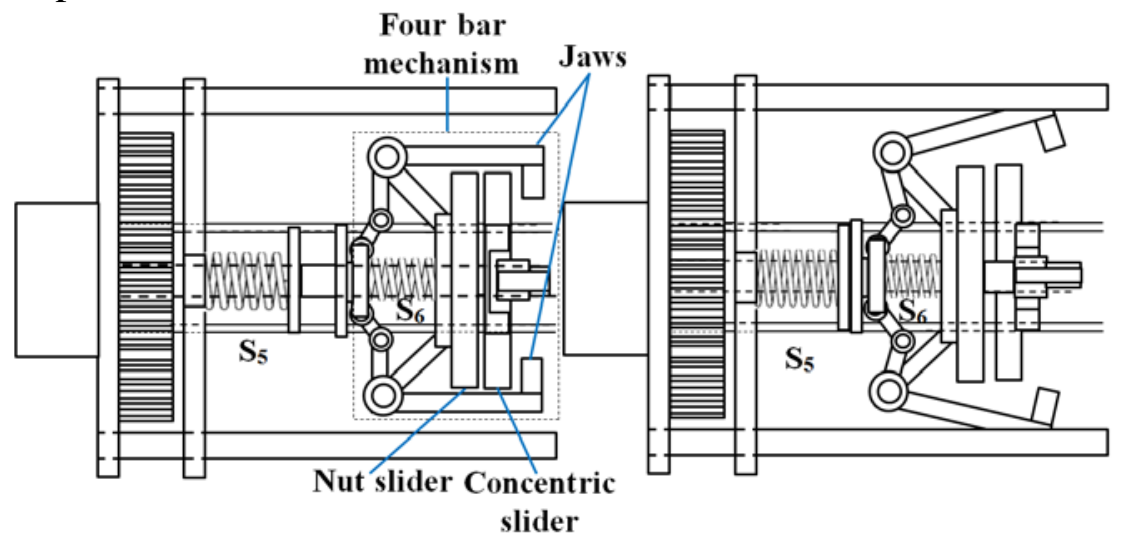

(a) Locked condition (b) Unlocked condition

Figure 5. Switching from motor based control to spring assisted force

After opening of the lock, the nut slider and concentric slider are detached from each other and the joint rotation is not under electric motor control. In this phase, patients are free to control their own movements and $S_{2}$ provides assistive force to help rotate the elbow joint (Fig. 6). The joint is torque balanced at every configuration due to the spring force and only a small effort is required from patients for lifting up any load or the forearm loads against gravity. Higher assistive force reduces the effort of users to reach a full joint rotation during flexion. The same assistive force opposes the forearm freefall during extension. In this way, the assistive force balances the arm weight and slows down the joint movement to achieve full extension. The assistive force during rehabilitation should be adaptable for different load under gravity. The structural part of the exoskeleton to provide variable gravity compensation consists of two torsional springs $\left(\mathrm{S}_{7}\right.$ and $\mathrm{S}_{8}$ ), one compression spring $\left(\mathrm{S}_{1}\right)$, one small cylindrical rod $\left(\mathrm{CR}_{1}\right)$, one small rectangular slider $\left(\mathrm{SL}_{1}\right)$ and two rectangular plates $\left(\mathrm{RP}_{1}\right.$ and $\left.\mathrm{RP}_{2}\right) . \mathrm{SL}_{1}$ is concentric to $\mathrm{CR}_{1}$ which is attached to the base plate. The range of spring force provided by $\mathrm{S}_{2}$ can be amplified by changing the span of displacement. $\mathrm{RP}_{1}$ and $\mathrm{RP}_{2}$ are connected to $\mathrm{SL}_{1}$ using $\mathrm{S}_{7}$ and $\mathrm{S}_{8}$ on both sides in such a way that these plates can rotate about the axis of these 
torsional springs (see the magnified view below). $\mathrm{RP}_{1}$ and $\mathrm{RP}_{2}$ have been used to maintain the force balancing condition during rehabilitation to provide a constant supply of assistive force. $\mathrm{CR}_{1}$ has a rectangular channel to provide a guiding path to $\mathrm{SL}_{1}$. The guiding path has two mechanical restrictions for controlling the movement of $\mathrm{SL}_{1}$ within a particular range. This is the region where different spring force can be generated. The role of $S_{1}$ is to restore the whole setup to its original position once released.

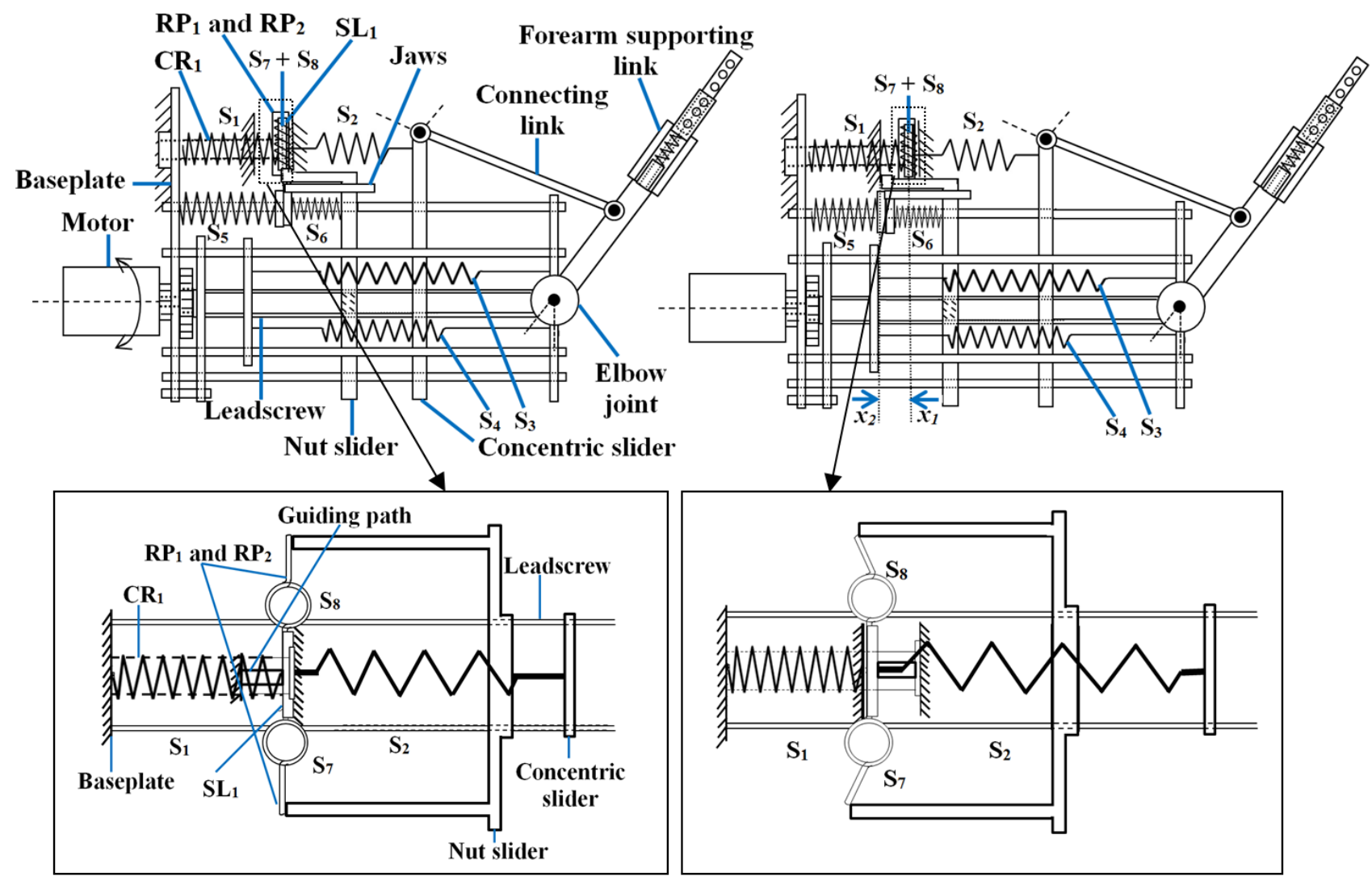

Figure 6. Spring configurations during assistive force mode

At the initial condition of self-initiated joint movement, the front end of $S_{2}$ is fixed which allows a fixed range of spring force. To increase the spring force dynamically, the front-end of $S_{2}$ is shifted backward near the baseplate; the extended part of nut slider has been utilized for this purpose. The backward movement of the nut slider in this region pushes $R_{1}$ and $R P_{2}$ connected to $S_{7}$ and $S_{8}$. The stiffness of $S_{7}$ and $S_{8}$ is high enough to be deflected by a small force, as a result, the whole arrangement connected to $\mathrm{SL}_{1}$ will move backward along with the nut slider. Due to the torsional stiffness, $S_{7}$ and $S_{8}$ create an opposing torque which is equalized by the reaction force from the nut slider during the movement. The second mechanical restriction on the guiding path does not allow $\mathrm{SL}_{1}$ to move further in the backward direction. This is the position where the mechanism can develop maximum assistive force at the joint using $S_{2}$. Therefore, further pressure from the nut slider will put $\mathrm{S}_{7}$ and $\mathrm{S}_{8}$ beyond their limit and $\mathrm{RP}_{1}$ and $\mathrm{RP}_{2}$ are deflected to come out from the range of nut slider. Because of the stiffness property of $\mathrm{S}_{1}$, $\mathrm{SL}_{1}$ will come to its initial position with all its arrangement.

\subsection{Resistance based rehabilitation (Third region)}

In this region, the exoskeleton provides a resistive force to the joint to restrict its motion which is achieved by changing the elbow joint stiffness. Two pairs of extension springs $\left(S_{3}\right.$ and $\left.S_{4}\right)$ are connected in parallel to the end of elbow joint on both sides and can slide on two solid parallel rods. Backward movement of the nut slider beyond the region $\left(x>x_{2}\right)$ will stretch both $S_{3}$ and $S_{4}$ resulting in higher joint stiffness (Fig. 7). Two linear springs $\left(S_{3}\right.$ and $\left.S_{4}\right)$ of different stiffness have been used in this design. 


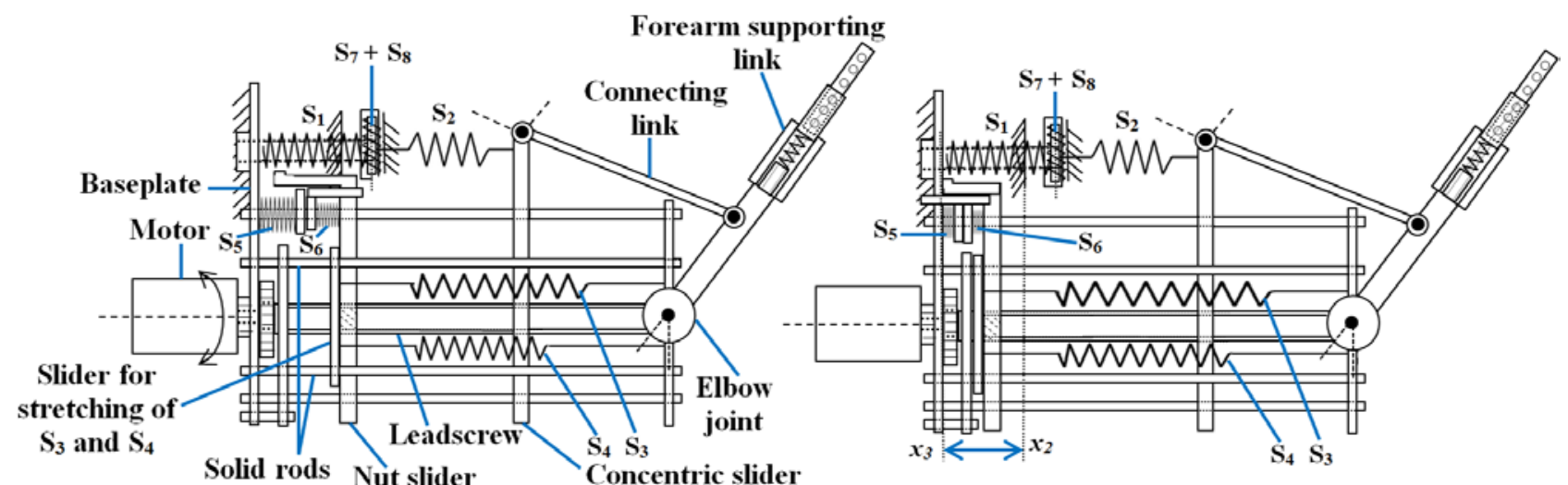

Figure 7. Spring configuration in resistive force mode for variable joint stiffness

The mechanism of the exoskeleton has been improved from other designs to get more flexibility such that a universal joint is used to replace the normal revolute joint for elbow movement so that the forearm is not fixed during flexion and extension. The universal joint provides a slight lateral movement of $\left( \pm 5^{\circ}\right)$. Out of the two degrees of freedom possessed by the universal joint, active one is responsible for flexion-extension of the elbow whereas the passive joint supports flexibility in the transverse plane during joint rotation. Another universal joint is used at the junction between the leadscrew slider and the connecting link to support the joint flexibility. The user's forearm is attached to the exoskeleton's forearm using cuff and straps. The forearm supporting link has discrete holes to fit different arm lengths. To maintain the alignment of the centre of rotation between the exoskeleton and user, the forearm has a passive translational joint with a compression spring whose length is varied to match the forearm length of the user as shown in Fig. 3.

\section{Torque analysis for motor control}

In the first region, the motor torque required to actuate the elbow joint is equivalent to the torque needed to overcome the frictional force created between the leadscrew and the nut slider. Due to the slider-crank mechanism, the elbow joint is actuated by pulling the connecting link (Fig. 8).

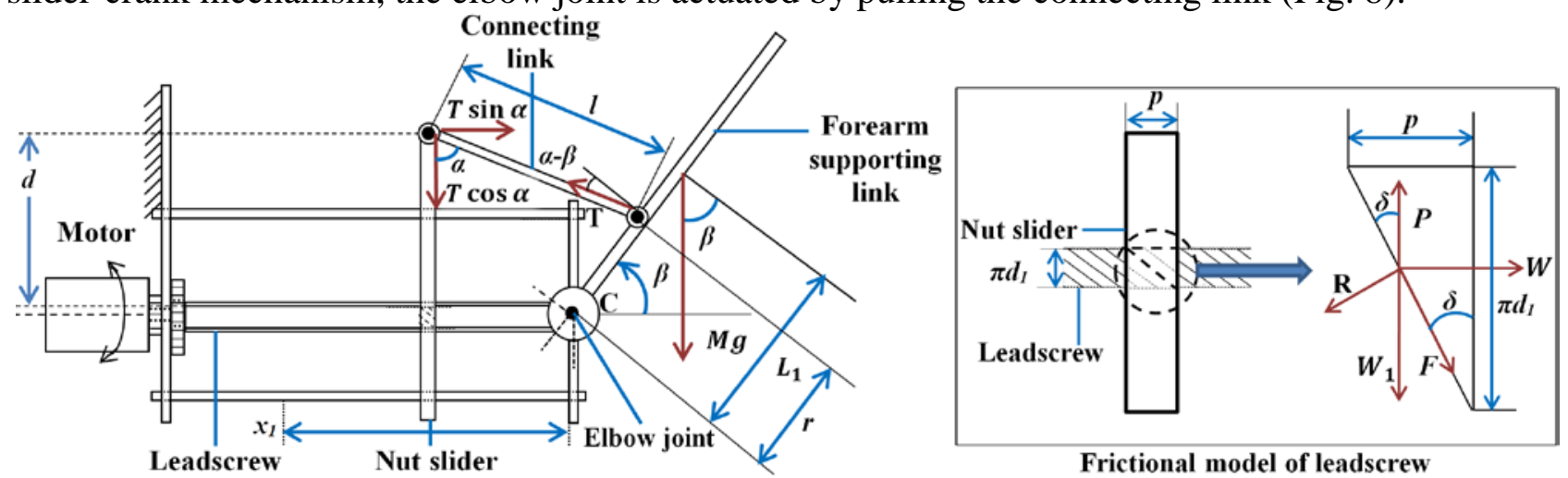

Figure 8. Mechanism analysis during electric motor control

The required force $(T)$ for lifting up the weight of the forearm is given by

$T=\frac{M g L_{1} \cos \beta}{r \cos (\alpha-\beta)}$

Where, $M$ - Mass of the forearm and the supporting link

$L_{1}$ - Distance from the elbow joint to the centre of gravity of forearm and the supporting link

$\beta$ - Elbow joint angle

$\alpha$ - Angle made by the connecting link and nut slider

$r$ - Length of the crank

$g$ - Acceleration due to gravity

From the model of the leadscrew (Fig. 8), it can be shown, $\tan \delta=\frac{p}{\pi d_{1}}$

Where $p$ - Pitch of the leadscrew, $d_{1}$ - Diameter of the leadscrew and $\delta$ - Lead angle of leadscrew 
$P$ is effort applied to the screw to lift the load. Taking the force equilibrium (Fig. 8),

We have, $P \cos \delta=W_{1} \cos \delta+W \sin \delta+F \quad$ [Where $T \cos \alpha=W_{1}$ and $T \sin \alpha=W$ ]

Frictional force $(F)$ during motion is

$F=\mu R_{N}=\mu\left(W_{1} \sin \delta-W \cos \delta-P \sin \delta\right)$ [where $\mu=$ coefficient of friction]

After substituting the value of $F$ and $\mu=\tan \varphi$ ( $\varphi$ is friction angle)] in Eq. (4), we get

$P=W_{1}+W \tan (\delta-\varphi)$

Putting the value of $W$ and $W_{1}$, torque $(\tau)$ required for overcoming the friction of the leadscrew is $\tau=P \times \frac{d_{1}}{2}=\frac{T(\cos \alpha+\sin \alpha \tan (\delta-\varphi)) d_{1}}{2}$

Putting the value of $T$ from Eq. (2), the final equation for required motor torque $(\tau)$ of the exoskeleton is $\tau=\frac{M g L_{1} \cos \beta(\cos \alpha+\sin \alpha \tan (\delta-\varphi)) d_{1}}{2 r \cos (\alpha-\beta)}$

The relation between $\alpha$ and $\beta$ can be derived from Fig. 8 as $\alpha=\cos ^{-1}\left(\frac{d-r \sin \beta}{l}\right)$

If motor is placed directly on the joint, the required motor torque is $\tau^{\prime}=M g L_{1} \cos \beta$

Fig. 9 shows the required motor torque in two configurations which is significantly reduced for the proposed exoskleton.

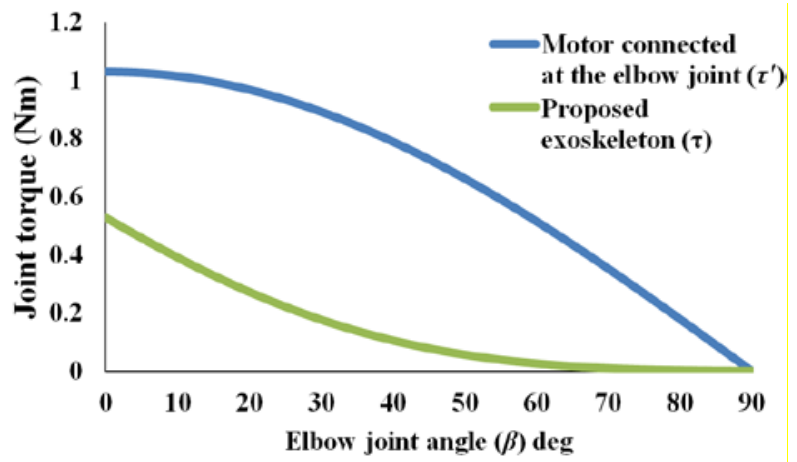

Figure 9. Comparison of the motor torque in two configurations

\section{Selection of springs and their stiffness calculation}

Since the linear springs $\left(S_{1}, S_{2}, S_{3}, S_{4}, S_{5}\right.$ and $\left.S_{6}\right)$ and torsional springs $\left(S_{7}\right.$ and $\left.S_{8}\right)$ are used in the design either for providing the spring force or switching from one stage of rehabilitation to another, therefore, the stiffness of all springs must be determined for the exoskeleton design.

\subsection{Stiffness of $S_{1}$}

The function of $S_{1}$ is to restore the position of the front-end of $S_{2}$ at the end of the assistive force region, therefore, the stiffness of $S_{1}$ needs to be high enough to overcome the frictional force between $\mathrm{SL}_{1}$ (along with the all other components connected to it) and $\mathrm{CR}_{1}$, see Fig. 10.

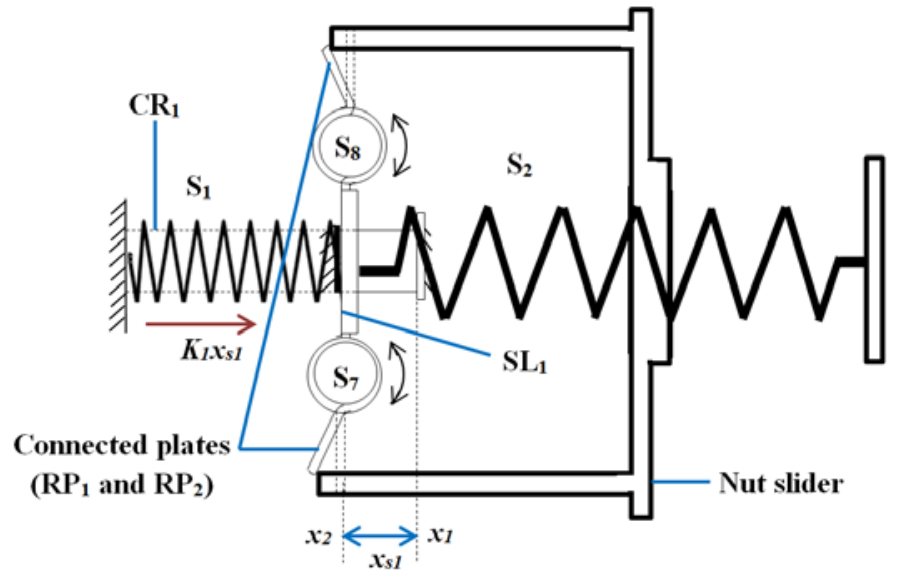

Figure 10. $\mathrm{S}_{1}$ in fully compressed state 
Therefore, $K_{1} x_{s 1}>\mu m g$

Where $K_{1}$ - Stiffness of $S_{1}$

$x_{s 1}$ - Displacement covered by $S_{1}$ in fully compressed position

$\mu$ - Coefficient of friction between $\mathrm{SL}_{1}$ and $\mathrm{CR}_{1}$

$m$ - Weight of the assembly connected to $\mathrm{SL}_{1}$

$g$ - Acceleration due to gravity

Based on the frictional property of $\mathrm{SL}_{1}$ and $\mathrm{CR}_{1}$ and mass of $\mathrm{SL}_{1}$, frictional force can be determined, from where $K_{1}$ can be estimated. $\mathrm{S}_{1}$ produces maximum force when it is fully compressed $\left(x=x_{2}\right)$ by the nut slider.

\subsection{Stiffness of $S_{2}$}

Spring force of $\mathrm{S}_{2}$ is mainly responsible for assisting the elbow movement in the second region (Fig. 11). In the exoskeleton, $S_{2}$ will be extended for sharing the required torque used to rotate the joint against gravity.

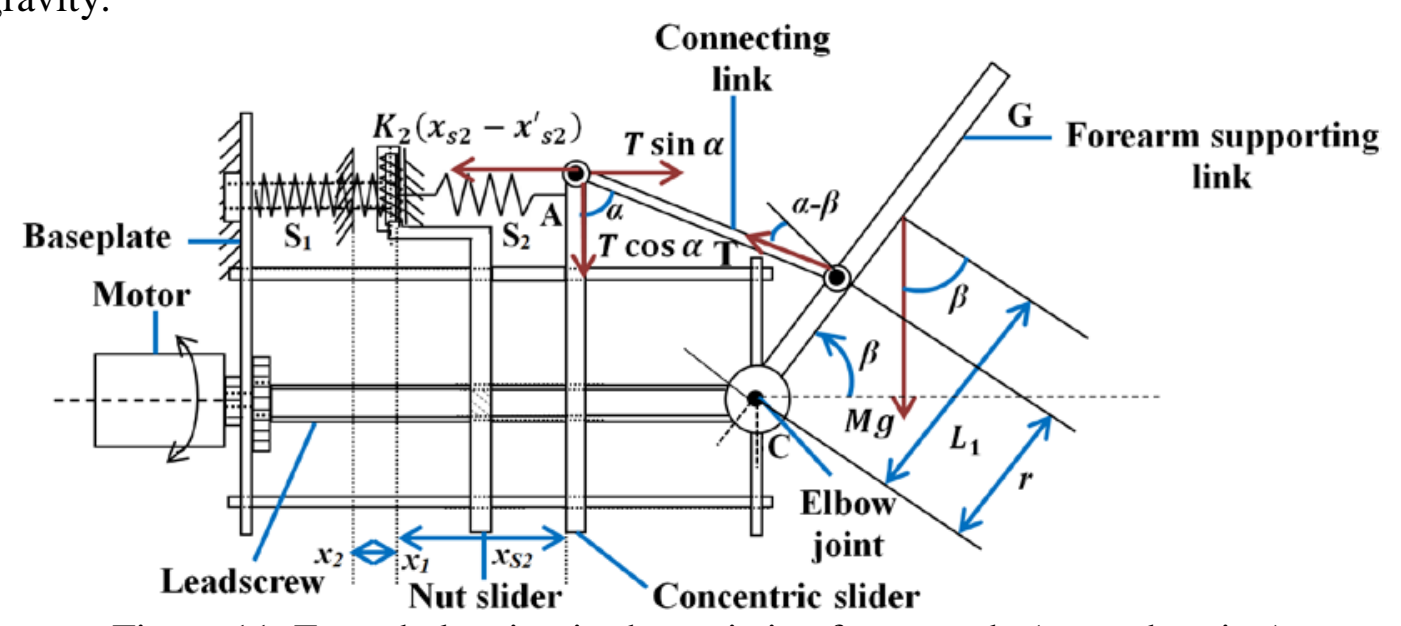

Figure 11. Force balancing in the assistive force mode (second region)

As linear bearings are used at the sliding contact between the concentric slider and leadscrew, the frictional force during motion is considered negligible compared to the elbow actuation force and is not taken into account.

The assistive force provided by $\mathrm{S}_{2}$ is $f_{s 2}=K_{2}\left(x_{s 2}-x_{s 2}^{\prime}\right)$

Where $K_{2}$ - Stiffness of $S_{2}, x_{s 2}$ - Displacement of $S_{2}, x_{s 2}^{\prime}$ - Free length of $S_{2}$.

Pulling force $(T)$ along the connecting link is same as it is shown during the electric motor control. Therefore, the value of $T$ is taken from Eq. (2). The only difference is that $S_{2}$ is taking the load instead of the motor.

Therefore, by equilibrating forces in Fig. 11, the stiffness of $S_{2}$ becomes

$K_{2}=\frac{M g L_{1} \cos \beta \sin \alpha}{r \cos (\alpha-\beta)\left(x_{s 2}-x \prime_{s 2}\right)}$

The displacement range of $S_{2}$ can be increased by pushing the nut slider backward towards the baseplate, thus providing more assistive force.

\subsection{Stiffness of $S_{3}$ and $S_{4}$}

The stiffness of $S_{3}$ and $S_{4}$ are used for changing the joint stiffness providing the resistive force (Fig. 12). The elbow joint stiffness is dependent on three springs $\left(S_{2}, S_{3}\right.$ and $\left.S_{4}\right)$. However, the spring parameters of $S_{2}$ are constant during the resistive force control, only the displacement of $S_{3}$ and $S_{4}$ is changed to create a variable joint stiffness at the elbow joint. 


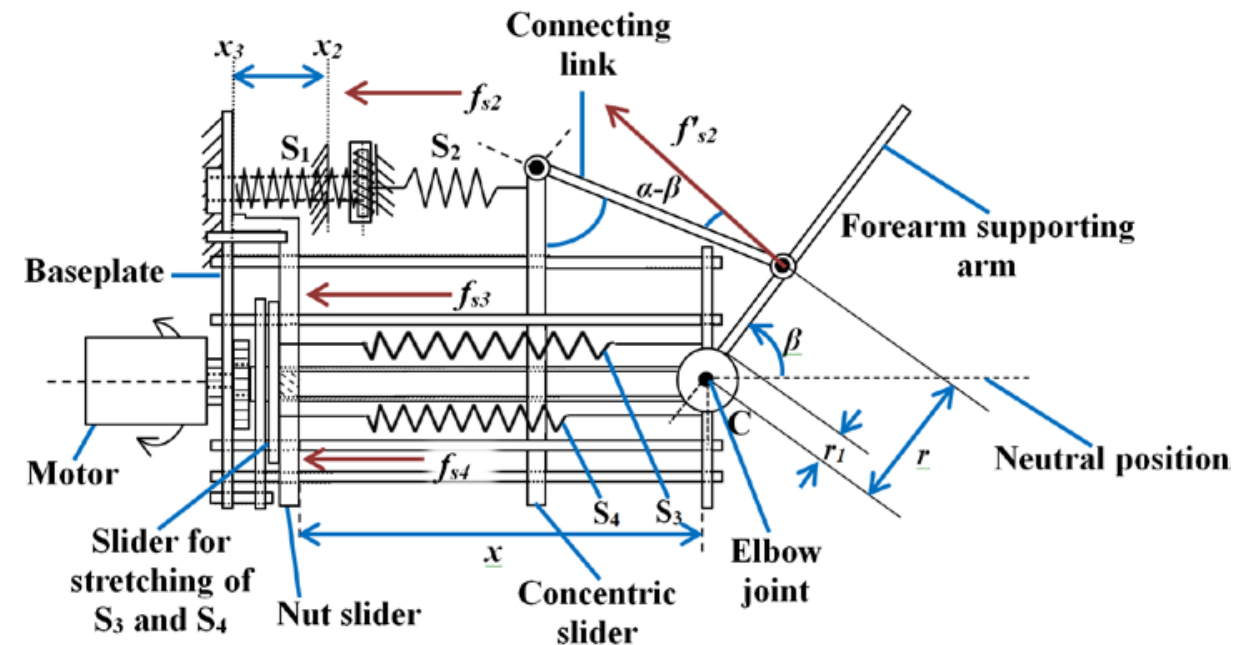

Figure 12. Elbow exoskeleton during variable joint stiffness control

The component of the spring force exerted by $\mathrm{S}_{2}$ about the point $\mathrm{C}$ is given by,

$f_{s 2}^{\prime}=\frac{K_{2}\left(x_{s 2}-x_{s 2}^{\prime}\right) \cos (\alpha-\beta)}{\sin \alpha}$

Spring force exerted by $\mathrm{S}_{3}$,

$f_{s 3}=K_{3}\left(x-r_{1} \beta-x_{s 3}^{\prime}\right)$

[Where $K_{3}$ - Stiffness of $S_{3}, x_{s 3}$ - Free length of $S_{3}$ ]

Spring force exerted by $\mathrm{S}_{4}$,

$f_{s 4}=K_{4}\left(x+r_{1} \beta-x^{\prime}{ }_{s 4}\right)$

[Where $K_{4}$ - Stiffness of $\mathrm{S}_{4}, X^{\prime}{ }^{\prime}$ - Free length of $\mathrm{S}_{4}$ ]

Therefore the joint stiffness of the elbow joint is the torsional stiffness $K$ ' which is given by

$K^{\prime}=\frac{\tau}{\beta}=\frac{r_{1}\left(f_{s 4}-f_{s 3}\right)}{\beta}-\frac{r f^{\prime}{ }_{s 2}}{\beta}$

Where $r_{1}$ - Radius of the pulley connected at the elbow joint

Two pairs of $S_{3}$ and $S_{4}$ are connected in this mechanism; therefore, the force exerted by both springs will be doubled. Substituting the value of $f_{s 2}, f_{s 3}$ and $f_{s 4}$ in Eq. (17), the elbow joint stiffness is given by

$K^{\prime}=\frac{2 r_{1}\left\{\left(K_{4}-K_{3}\right) x-\left(K_{4} x \prime_{s 4}-K_{3} x \prime_{s 3}\right)\right\}-\frac{K_{2} r\left(x_{s 2}-x \prime_{s 2}\right) \cos (\alpha-\beta)}{\sin \alpha}}{\beta}+2 r_{1}^{2}\left(K_{4}+K_{3}\right)$

The joint stiffness variation is shown in Fig. 13.

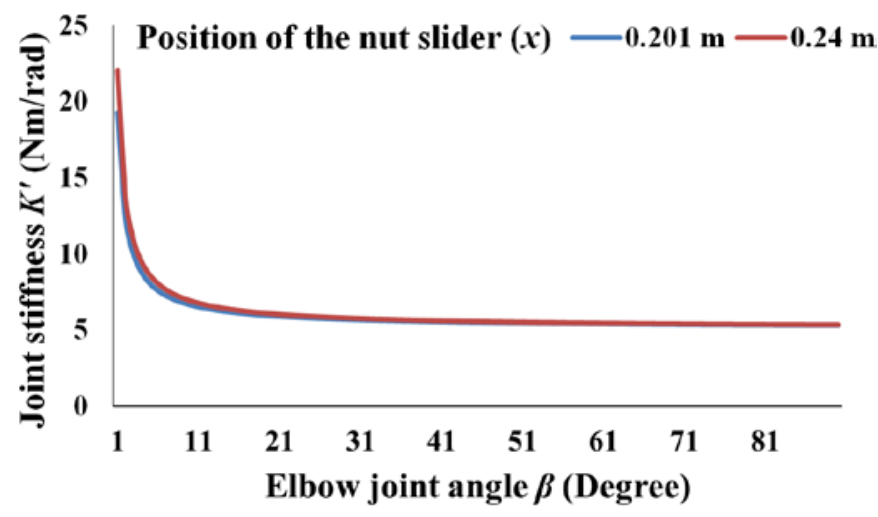

Figure 13. Elbow joint stiffness variation for different position of the nut slider

\subsection{Stiffness of $S_{5}$ and $S_{6}$}

The stiffness of both compression springs $\left(S_{5}\right.$ and $\left.S_{6}\right)$ used for locking operation is equally important in switching operation between first and second region. The ratio of the stiffness of $\mathrm{S}_{5}$ and $\mathrm{S}_{6}$ depends on the construction parameters of the locking mechanism (Fig. 14). 


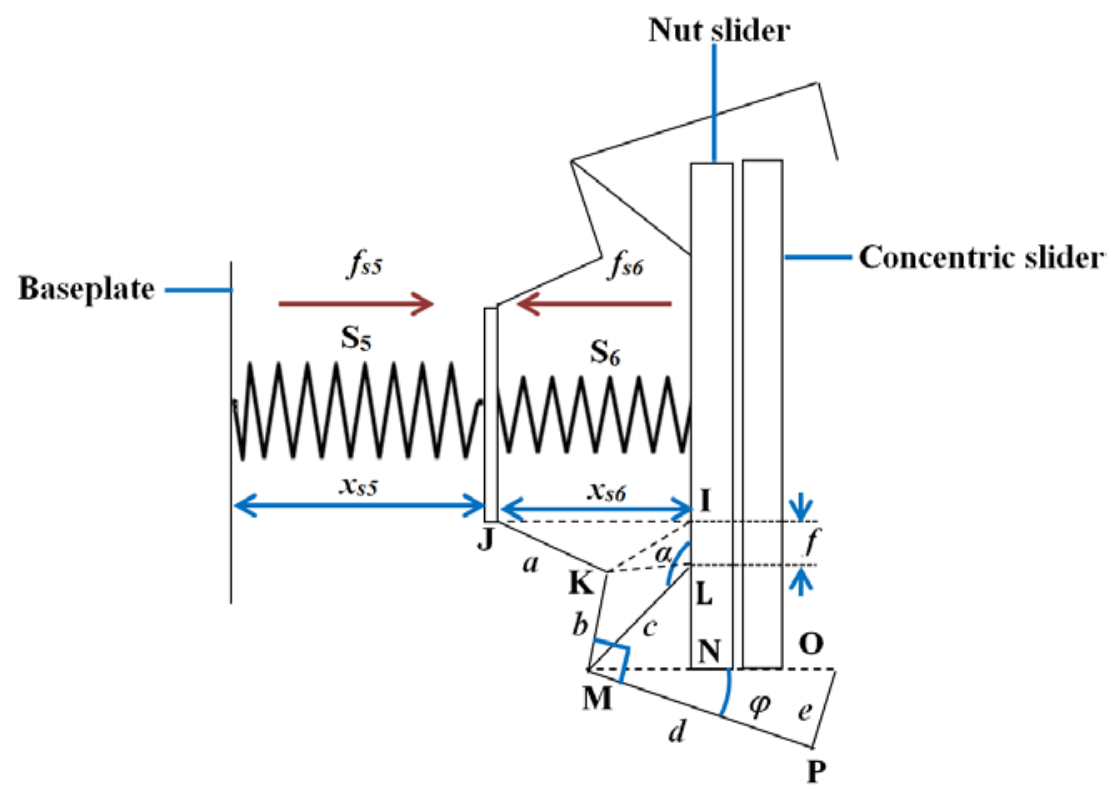

Figure 14. Force balancing during the unlocked condition

In the locking mechanism, the right-handed jaw KMPO is shown. The upper-end position of the jaw O should be outside of the region covered by these sliders (shown in dotted line OM). It is clear that these two jaws need to rotate a minimum angle $\varphi$ about point $\mathrm{M}$ to unlock the concentric slider from the locking range. Here, $\triangle \mathrm{OMP}$ is a right-angled triangle. Therefore, the required angle $\varphi$ for unlocking concentric slider from the jaw is

$$
\varphi=\tan ^{-1} \frac{e}{d}
$$

Length of the solid links used in this mechanism are $a, b, c, d, e$ and $f . \angle \mathrm{MLI}$ and $\angle \mathrm{KMP}$ are also a part of the structure. As the values of $e$ and $d$ are constant, the value of $\varphi$ is defined for the unlocking condition. To achieve the angle, $\mathrm{S}_{6}$ needs to move a particular distance which can be derived by the geometrical parameters.

The locking mechanism can function successfully if it satisfies the following condition, $K_{5}$ » $K_{6}$ [Where $K_{5}$ - Stiffness of $S_{5}, K_{6}$ - Stiffness of $S_{6}$ ] which means, $x_{55}$ « $x_{s 6}$

At the time of opening the lock, both springs will be in equilibrium which means force exerted by $\mathrm{S}_{5}$ and $\mathrm{S}_{6}$ will be the same at that position.

Therefore, $f_{5}=f_{6}$

i.e. $K_{5} x_{s 5}=K_{6} x_{s 6}$

$K_{5}=\frac{K_{6} x_{s 6}}{x_{s 5}}$

From Fig. 14, cos $\angle \mathrm{JIK}=\frac{x_{s 6}{ }^{2}+\mathrm{KI}^{2}-a^{2}}{2 x_{s 6} \mathrm{KI}}$

$\cos \left(90^{\circ}-\angle \mathrm{KIL}\right)=\frac{x_{s 6^{2}+\mathrm{KI}^{2}-a^{2}}}{2 x_{s 6} \mathrm{KI}}$

Using trigonometric relation, it can be found that

$$
\mathrm{KI}^{2}=f^{2}+A_{1}-2 f \sqrt{A_{1}} \cos \left(\delta-\cos ^{-1}\left(\frac{B_{1}}{\sqrt{A_{1}}}\right)\right)
$$

and $\angle \mathrm{KIL}=\cos ^{-1}\left(\frac{f-\sqrt{A_{1}} \cos \left(\delta-\cos ^{-1}\left(\frac{B_{1}}{\sqrt{A_{1}}}\right)\right)}{f^{2}+A_{1}-2 f \sqrt{A_{1}} \cos \left(\delta-\cos ^{-1}\left(\frac{B_{1}}{\sqrt{A_{1}}}\right)\right)}\right)$

Where $A_{1}=b^{2}+c^{2}+2 b c \cos \left(\delta+\tan ^{-1} \frac{e}{d}\right)$

$B_{1}=c+b \cos \left(\delta+\tan ^{-1} \frac{e}{d}\right)$ 
After calculating the value of $x_{56}, x_{55}$ and putting in Eq. (20), we get relationship between $K_{5}$ and $K_{6}$.

The spring $\left(\mathrm{S}_{6}\right)$ associated with the lock will experience a higher and opposite force from $\mathrm{S}_{5}$. After opening of the lock, $S_{6}$ cannot be compressed further due to the mechanical constraint thus exhibiting a constant force for the rest of the motion. However, due to the movement of nut slider in backward direction, $S_{5}$ will be further compressed with a higher spring force which helps to maintain the unlocked condition during rest of the range as shown Fig. 15.

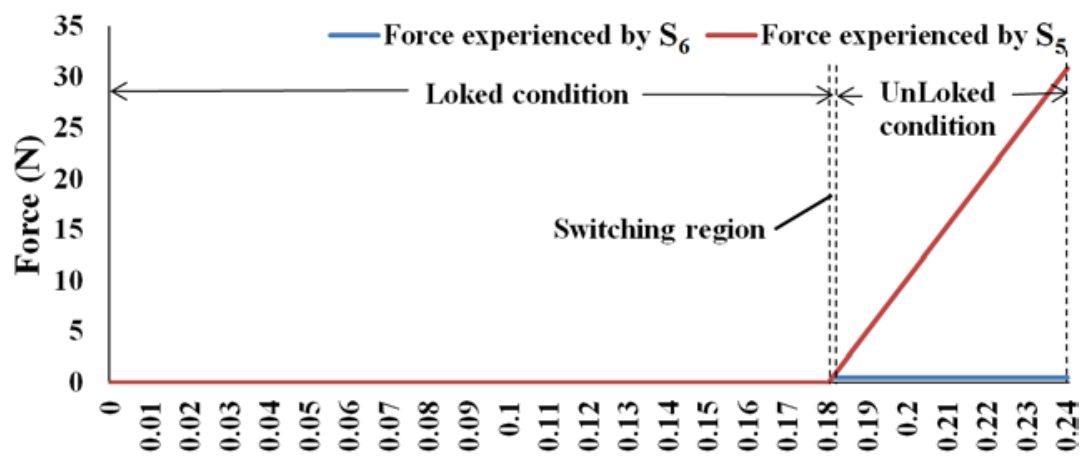

Position of nut slider $(x)(\mathrm{m})$

Figure 15. Force generation in two springs of the locking mechanism

\subsection{Stiffness of $S_{7}$ and $S_{8}$}

Fig. 16 shows the force balancing of the mechanism during the final stage of the assistive mode where both torsional springs $\mathrm{S}_{7}$ and $\mathrm{S}_{8}$ are at their maximum deflected position. The nut slider generates an equal and opposite force against two torsional springs $\left(S_{7}\right.$ and $S_{8}$ ) and balances the forces generated by $S_{1}$ and $S_{2}$. Both $S_{7}$ and $S_{8}$ have equal stiffness as they are structurally the same.

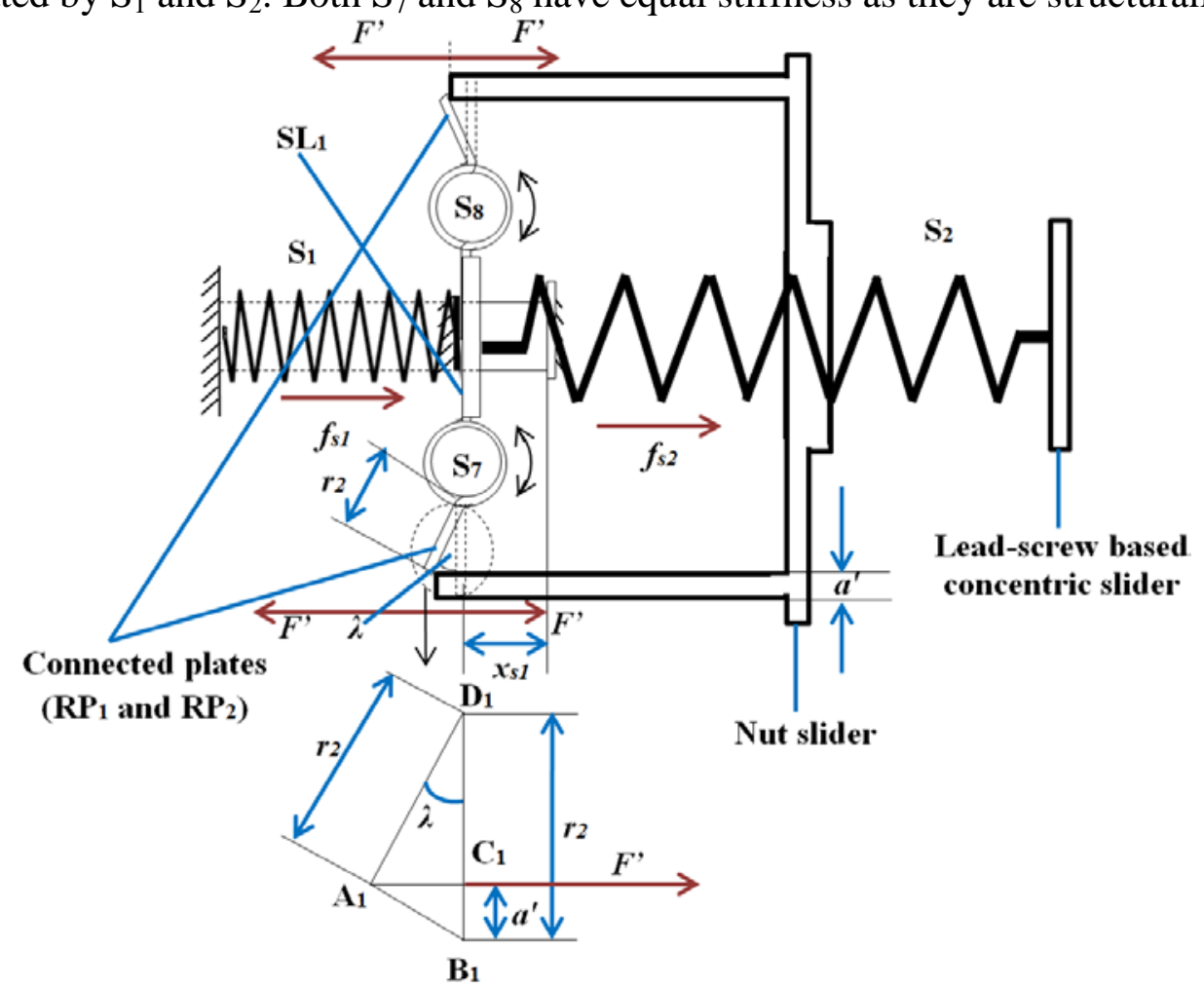

Figure 16. Force balancing of the mechanism at the final stage of assistive force mode

From Fig. 16, it can be derived that,

$358 \lambda=\cos ^{-1}\left(1-\frac{a \prime}{r_{2}}\right)$

359 Where $a^{\prime}$ - Width of the extension part of the nut slider, $r_{2}$ - Length of the plates $\left(\mathrm{RP}_{1}\right.$ and $\left.\mathrm{RP}_{2}\right)$

360 Force produced by $\mathrm{S}_{1}, f_{s 1}=K_{1} x_{s 1} \quad$ [Where $K_{1}$ - Stiffness of $\mathrm{S}_{1}$ ] 
From Fig. $16, \tau^{\prime}=K_{7} \lambda$

Where $\lambda$ - Angle made $\mathrm{RP}_{1}$ and $\mathrm{RP}_{2}$ at maximum deflected position

$\tau^{\prime}$ - Torque created by $\mathrm{S}_{7}$ and $\mathrm{S}_{8}$

$F^{\prime} r_{2} \cos \lambda=K_{7} \lambda$ Where $F^{\prime}$ - Reaction force by $\mathrm{S}_{7}$ and $\mathrm{S}_{8}$

Therefore, $F^{\prime}=\frac{K_{7} \lambda}{r_{2} \cos \lambda}$

As $\mathrm{S}_{7}$ and $\mathrm{S}_{8}$ maintain the force in equilibrium,

Putting the value of $f_{s 1}$ (from Eq. (25)), $f_{s 2}$ (from Eq. (26)) and $F^{\prime}$ (from Eq. (27)) in Eq. (28), we

have $K_{7}=\frac{r_{2} \cos \lambda\left(K_{1} x_{s 1}+K_{2}\left(x_{s 2}+x_{s 1}-x_{s 2}\right)\right)}{2 \lambda}$

After putting the value of $\lambda$ (taken from Eq. (24)), the value of $K_{7}$ will be,

$\mathrm{K}_{7}=\frac{\left(r_{2}-a^{\prime}\right)\left(K_{1} x_{s 1}+K_{2}\left(x_{s 2}+x_{s 1}-x^{\prime} s 2\right)\right)}{2 \cos ^{-1}\left(1-\frac{a^{\prime}}{r_{2}}\right)}$

Based on the above design considerations, a functional prototype has been developed to establish the working principle of the exoskeleton. All mechanical components have been manufactured using 3D printer. The prototype of the elbow exoskeleton is shown in Fig. 17 along with its specifications. All the sliding contacts have been developed with bearing to reduce the frictional loss during motion. The prototype performs as per the requirements of the three stages of rehabilitation.

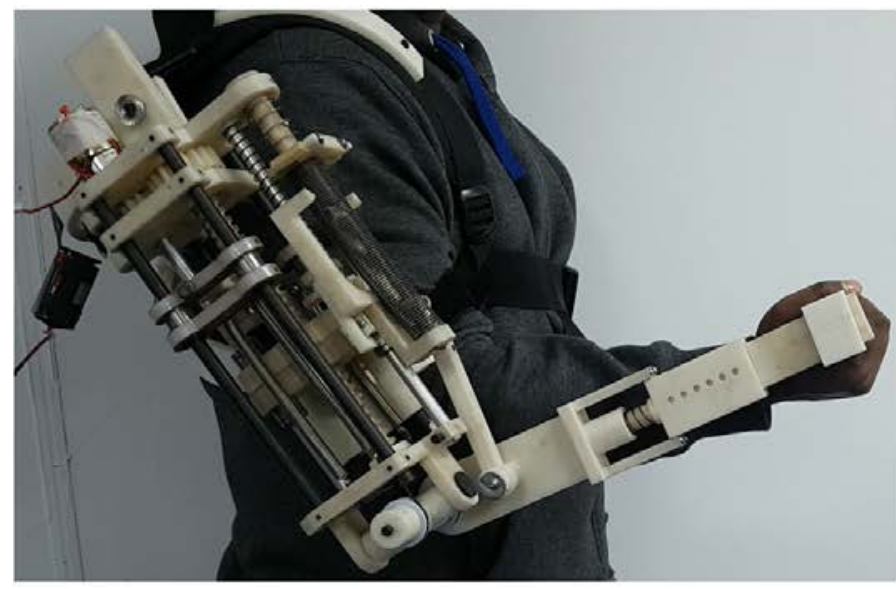

\begin{tabular}{|c|c|}
\hline \multirow{2}{*}{ Material } & $\begin{array}{c}\text { ABS (Acrylonitrile butadiene } \\
\text { styrene) }\end{array}$ \\
\hline Upper arm dimension & $0.35 \mathrm{~m} \times 0.15 \mathrm{~m} \times 0.17 \mathrm{~m}$ \\
\hline Forearm dimension & $0.40 \mathrm{~m} \times 0.02 \mathrm{~m} \times 0.06 \mathrm{~m}$ \\
\hline Weight & $1.8 \mathrm{~kg}$ \\
\hline \multirow{4}{*}{ Motor specification } & Model-DFROBOT ZYTD520 \\
\cline { 2 - 2 } & Operating voltage-12V DC \\
\cline { 2 - 2 } & RPM-50 RPM \\
\cline { 2 - 2 } & Maximum torque-4.6 Nm \\
\cline { 2 - 2 } & Weight-0.210 kg \\
\hline Gear material & Power -5 W \\
\hline Spring material & Nylon-101 \\
\hline Linear Ball Bearing & $\begin{array}{c}\text { Model- Bosch Rexroth Linear } \\
\text { Ball Bearing R060204010 }\end{array}$ \\
\hline Axial Ball bearing & HCH 62022 \\
\hline
\end{tabular}

Figure 17. Prototype of the elbow exoskeleton with specifications

\section{Conclusions}

An innovative mechanism of the elbow exoskeleton has been developed which can accommodate three modes of rehabilitation for different stages after stroke. In this design, we have attempted to achieve the multistage post-stroke rehabilitation at mechanical level so that the device can be finetuned to user's requirements. Full design details of the elbow exoskeleton have been presented together with parametric relations for component selection. These design parameters can be tailored to suit any user specific requirements. A prototype device has been developed to prove the principle. For most exoskeletons, the motor torque is varied depending on the dynamics of the model and patient's requirement whereas in this exoskeleton the position of the nut-slider can produce different exercise modes either under motor control or in assistive or resistive modes. The mechanism can change the amount of assistive and resistive force by simply changing the position of the slider. Such arrangement in a single structure offers flexibility to patients to select a particular type of exercise. 
During the assistive and resistive modes only the spring force is used without engaging any active actuator therefore the energy source is only used during the motor operation. The switching mechanism safeguards users by restricting the reachable joint angle to the anatomical limit during motor control mode. If the nut sliver goes up to the end of first rehabilitation region due to motor rotation, the lock will be actived till the joint takes its maximum anatomical limit. Position of the nut slider beyond the switching point will automatically open the lock, releasing the joint control from motor and transfer it to the user, therefore providing safety and functionality at the same time.

\section{References}

[1] Feigin, V. L., Forouzanfar, M. H., Krishnamurthi, R., Mensah, G. A., Connor, M., Bennett, D. A., Moran, A. E., Sacco, R. L., Anderson, L., and Truelsen, T., 2014, "Global and regional burden of stroke during 1990-2010: findings from the Global Burden of Disease Study 2010," The Lancet, 383(9913), pp. 245-255.

[2] Saka, Ö., McGuire, A., and Wolfe, C., 2009, "Cost of stroke in the United Kingdom," Age and ageing, 38(1), pp. 2732.

[3] Lo, A. C., Guarino, P. D., Richards, L. G., Haselkorn, J. K., Wittenberg, G. F., Federman, D. G., Ringer, R. J., Wagner, T. H., Krebs, H. I., and Volpe, B. T., 2010, "Robot-assisted therapy for long-term upper-limb impairment after stroke," New England Journal of Medicine, 362(19), pp. 1772-1783.

[4] Manna, S. K., and Dubey, V. N., 2016, "Upper arm exoskeleton -what specifications will meet users' acceptability? ," Robotics: New Research D. G. Fisher, ed., Nova Science Publisher, pp. 123-169.

[5] Proietti, T., Crocher, V., Roby-Brami, A., and Jarrassé, N., 2016, "Upper-limb robotic exoskeletons for neurorehabilitation: a review on control strategies," IEEE Reviews in Biomedical Engineering, 9, pp. 4-14.

[6] Pineda-Rico, Z., de Lucio, J. A. S., Martinez Lopez, F. J., and Cruz, P., 2016, "2121. Design of an exoskeleton for upper limb robot-assisted rehabilitation based on co-simulation," Journal of Vibroengineering, 18(5).

[7] Chonnaparamutt, W., and Supsi, W., 2016, "SEFRE: Semiexoskeleton Rehabilitation System," Applied Bionics and Biomechanics, 2016.

[8] Beer, R. F., Naujokas, C., Bachrach, B., and Mayhew, D., "Development and evaluation of a gravity compensated training environment for robotic rehabilitation of post-stroke reaching," 2008, Proc. 2nd IEEE RAS \& EMBS International Conference on Biomedical Robotics and Biomechatronics, IEEE, pp. 205-210.

[9] Kolar, P., 2014, Clinical rehabilitation, Alena Kobesová.

[10] Jarrassé, N., Proietti, T., Crocher, V., Robertson, J., Sahbani, A., Morel, G., and Roby-Brami, A., 2014, "Robotic exoskeletons: a perspective for the rehabilitation of arm coordination in stroke patients," Frontiers in Human Neuroscience, 8, p. 947.

[11] Wolbrecht, E. T., Chan, V., Reinkensmeyer, D. J., and Bobrow, J. E., 2008, "Optimizing compliant, model-based robotic assistance to promote neurorehabilitation," IEEE Transactions on Neural Systems and Rehabilitation Engineering, 16(3), pp. 286-297.

[12] Maciejasz, P., Eschweiler, J., Gerlach-Hahn, K., Jansen-Troy, A., and Leonhardt, S., 2014, "A survey on robotic devices for upper limb rehabilitation," Journal of Neuroengineering and Rehabilitation, 11(1), p. 3.

[13] Bhadane, M., Liu, J., Rymer, W. Z., Zhou, P., and Li, S., 2016, "Re-evaluation of EMG-torque relation in chronic stroke using linear electrode array EMG recordings," Scientific Reports, 6, p. 28957.

[14] Marchal-Crespo, L., and Reinkensmeyer, D. J., 2009, "Review of control strategies for robotic movement training after neurologic injury," Journal of Neuroengineering and Rehabilitation, 6(1), p. 20.

[15] Marcheschi, S., Salsedo, F., Fontana, M., and Bergamasco, M., "Body extender: whole body exoskeleton for human power augmentation," Proc. 2011 IEEE International Conference on Robotics and Automation (ICRA), IEEE, pp. 611616.

[16] Manna, S. K., and Dubey, V. N., 2018, "Comparative study of actuation systems for portable upper limb exoskeletons," Medical Engineering \& Physics, 60, pp. 1-13.

[17] Housman, S. J., Le, V., Rahman, T., Sanchez, R. J., and Reinkensmeyer, D. J., "Arm-training with T-WREX after chronic stroke: preliminary results of a randomized controlled trial," Proc. IEEE 10th International Conference on Rehabilitation Robotics, 2007, IEEE, pp. 562-568.

[18] Sanchez, R., Reinkensmeyer, D., Shah, P., Liu, J., Rao, S., Smith, R., Cramer, S., Rahman, T., and Bobrow, J., "Monitoring functional arm movement for home-based therapy after stroke," Proc. 26th Annual International Conference of the IEEE Engineering in Medicine and Biology Society, 2004, IEEE, pp. 4787-4790.

[19] Crea, S., Cempini, M., Moisè, M., Baldoni, A., Trigili, E., Marconi, D., Cortese, M., Giovacchini, F., Posteraro, F., and Vitiello, N., "A novel shoulder-elbow exoskeleton with series elastic actuators," Proc. 2016 6th IEEE International Conference on Biomedical Robotics and Biomechatronics (BioRob), IEEE, pp. 1248-1253.

[20] Van Ham, R., Sugar, T. G., Vanderborght, B., Hollander, K. W., and Lefeber, D., 2009, "Compliant actuator designs," IEEE Robotics \& Automation Magazine, 16(3). 